\title{
Cyclic stroke mortality variations follow sunspot patterns
}

\section{[version 1; peer review: 2 approved]}

\section{Stella Geronikolou (D1, Alexandros Leontitsis², Vasilis Petropoulos3, Constantinos Davos ${ }^{4}$, Dennis Cokkinos 5 , George Chrousos (iD 6}

\footnotetext{
${ }^{1}$ Clinical, Translational and Exp Surgery, Biomedical Research Foundation of the Academy of Athens, Athens, 11527, Greece 2University of Ioannina, Ioannina, Greece

${ }^{3}$ Research Center for Astronomy and Applied Mathematics, Academy of Athens, Athens, 11527, Greece

${ }^{4}$ Clinical, Translational and Exp Surgery, Biomedical Research Foundation of the Academy of Athens, Athens, 11527, Greece

${ }^{5}$ Clinical Translational and Exp Surgery, Biomedical Research Foundation of the Academy of Athens, Athens, 11527, Greece 6University Research Institute of Maternal and Child Health and Precision Medicine, UNESCO Chair on Adolescent Health Care, National and Kapodistrian University of Athens, Thivon and Levadeias, Athens, 11527, Greece
}

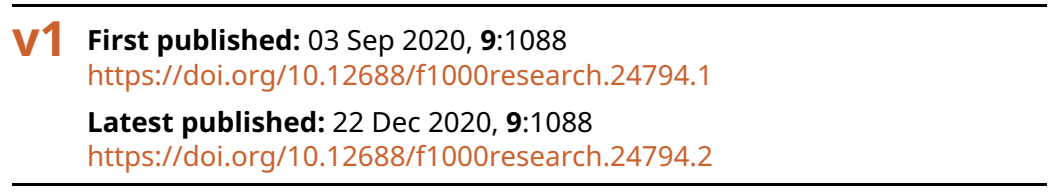

\section{Abstract}

Mapping time-structures is a burgeoning scientific field enriching the (P4) medicine models. Local evidence in Mediterranean populations is underinvestigated. The Censed stroke-related death events (D) in the largest East-Mediterranean port (Piraeus), during (1985-1989), when local population had diet and genetic homogeneity-been interrupted by the immigration into Greece in 1990s, and Sunspot numbers indexed by Wolf numbers (Rz) (1944-2004), were evaluated using Fast Fourier Analysis and Singular Spectrum Analysis in MATLAB. D were turned with fluctuations $>35 \%$ in Rz. A non-anthropogenic 6.8 days cycle was recognized. This study may be taken into consideration in future public health planning and chronotherapy evaluations.

\section{Keywords}

Sunspot numbers, Chronome, Stroke mortality, Singular Spectrum Approach, NCOR1, R1 interactome

\section{HEAL 1000}

This article is included in the HEAL1000 gateway.

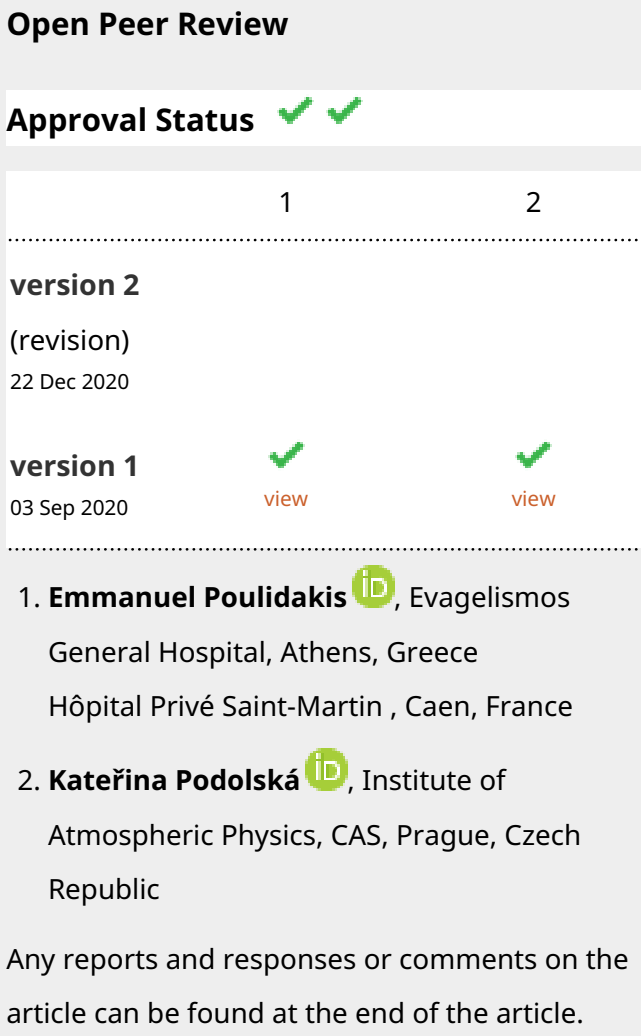


Corresponding author: Stella Geronikolou (sgeronik@gmail.com)

Author roles: Geronikolou S: Conceptualization, Data Curation, Formal Analysis, Investigation, Methodology, Resources, Writing Original Draft Preparation; Leontitsis A: Data Curation, Methodology, Software, Validation; Petropoulos V: Conceptualization, Validation; Davos C: Writing - Review \& Editing; Cokkinos D: Supervision, Validation, Writing - Review \& Editing; Chrousos G: Conceptualization, Supervision, Validation, Writing - Review \& Editing

Competing interests: No competing interests were disclosed.

Grant information: The author(s) declared that no grants were involved in supporting this work.

Copyright: ( 2020 Geronikolou S et al. This is an open access article distributed under the terms of the Creative Commons Attribution License, which permits unrestricted use, distribution, and reproduction in any medium, provided the original work is properly cited.

How to cite this article: Geronikolou S, Leontitsis A, Petropoulos V et al. Cyclic stroke mortality variations follow sunspot patterns [version 1; peer review: 2 approved] F1000Research 2020, 9:1088 https://doi.org/10.12688/f1000research.24794.1

First published: 03 Sep 2020, 9:1088 https://doi.org/10.12688/f1000research.24794.1 


\section{Introduction}

Strokes are the second leading cause of death and disability worldwide (Mozaffarian et al., 2015). Strokes share all of the recommended interventions for chronic noncommunicable disease (NCD): life-style modifications, including a low fat/ salt/sugar diet, moderate physical activity, discontinuation of smoking, sufficient sleep, and control of arterial blood pressure control, and, if necessary, pharmacologic therapy (Mozaffarian et al., 2015). In addition, risk factors such as chronic stress, underlying diseases, such as obesity, diabetes mellitus, chronic obstructive pulmonary disease, and renal insufficiency, as well as predisposing genetic factors, have been implicated in stroke morbidity and mortality (Malik \& Dichgans, 2018). The incidence and prevalence of stroke subtypes vary greatly, depending on ethnicity and country income. As stroke statistics fail to cover all etiologies, some remain unknown.

Stroke has been previously associated to solar activity (Halgberg et al., 2001; Otsuka et al., 2001; Stoupel et al., 1995; Stoupel et al., 1996). Such activity, as indexed by sunspot numbers, has been generally associated with health (Feigin et al., 2014; Halberg et al., 1998; Petropoulos \& Geronikolou, 2005). However, relevant chaos and trend analyses in local populations have been limited but strongly suggested (Reinberg et al., 2017).

Our aim was to investigate the dynamics and trends in the time series, to determine sunspot numbers vs. daily and monthly stroke deaths in synchronized periodicities with gradual time delays (chronomes), and to define a sunspot number threshold for presence of stroke mortality.

\section{Methods}

In this study we focused on monthly stroke mortality events between 1985 and 1989, based on the underlined cause of death data from the archives of Piraeus Civil Registry (Geronikolou \& Zikos, 1991)

The sunspot numbers were derived from the archives of measurements published by Solar Geographical Data. Sunspot Number, denoted $\mathrm{Rz}$ (Zurich number), is defined as: $R z=$ $\mathrm{K}(10 \mathrm{~g}+\mathrm{f})$, where $\mathrm{g}$ is the number of sunspot groups visible on the Sun, $\mathrm{f}$ represents the total number of individual spots visible; and $\mathrm{K}$ is an instrumental factor to take into account differences between observers and observatories.

The stroke death rate in Piraeus, was calculated over the formula (number of all deaths per year per 1000 people in June $30^{\text {th }}$, year $\mathrm{x}$ ). The overall death rate was calculated with the denominators provided by the 1981 census.

In the analysis of our short time series, we employed fast Fourier transform (FFT) analysis and the singular spectrum approach (SSA). Thus, we first performed a square root transformation of the sunspot time series. We subsequently analyzed the second time series of the stroke deaths using the SSA, to find the principal components that formulated it using principal component analysis (PCA). We applied Pearson correlation analysis to detect the coefficients of variation between the principal components of the sunspots and the strokes time series. All calculations were performed with MATLAB 7 software.

\section{Results}

We focused on monthly stroke deaths, based on all death events archived in the local Civil Registry (Geronikolou \& Zikos, 1991). There were 792 stroke deaths out of 4324 total deaths events distributed in the 60 months of the quinquennium (19851989) examined. Over $54 \%$ were women and over $61 \%$ occurred at ages over 69 years. The stroke death rate (stroke deaths in year $x$ /overall deaths in year $x \times 100$ ) was calculated as 17.668 in 1985, 20.089 in 1986, 19.372 in $1987,17.647$ in 1988 , and 15.531 in 1989. The overall death rate (all deaths in year $x$ /local population in year $x \times 100$ ) was 5.5 in 1985, 4.4 in 1986, 4.9 in 1987, 3.5 in 1988, and 3.7 in 1989.

The observed time series of both monthly and daily sunspot numbers and monthly and daily stroke death events in Piraeus between 1985 and 1989 are illustrated in Figure 1a. The PCA distinguished two principal components, as shown in Figure 1b: 6.8 and 20 days. The sunspot numbers observed (1944-2004) transformed to squared roots are described in Figure 1c. The singular values of the transformed sunspots time series showed that the noise plateau began at the $3^{\text {rd }}$ ordered singular value (Figure 1d). Thus, monthly sunspot numbers by squared root variation and their violent fluctuation of over $35 \%$ was correlated to monthly stroke mortality, establishing a negative correlation between the two time-series (sunspot numbers and deaths of strokes) (Figure 1b, d). FFT showed frequencies of 3.5 and 6.85 days.

Data on stroke deaths and sunspots by month are available as Underlying data (Geronikolou \& Leontitsis, 2020).

\section{Discussion}

Mapping time-structures is a rapidly growing scientific field, enriching P4 medicine (predictive, preventative, personalized, participatory medicine) models with chronotherapy aspects (Yan, 2015). Human biological clocks are intensively studied. They represent adaptive body mechanisms necessary to assist with homeostatic changes caused by solar activity disturbances. These mechanisms have not been extensively investigated in Mediterranean populations.

Chronic NCDs account for over $70 \%$ of early deaths worldwide, while stroke is the second leading cause of death and disability; the latter is associated with high expenses in health services, and constitutes a public health challenge (WHO, 2018). This challenge is progressively increasing, considering the large population migrations that take place on the planet because of ethnic conflicts, economic crises and climate changes. Stroke has been associated with various risk factors, such as lifestylerelated eating habits, tobacco and/or alcohol use, and decreased physical activity, underlying comorbidities, such as obesity, hypertension, dyslipidemia, diabetes mellitus type 2, lung and kidney failures, etc., as well as exposure to environmental 

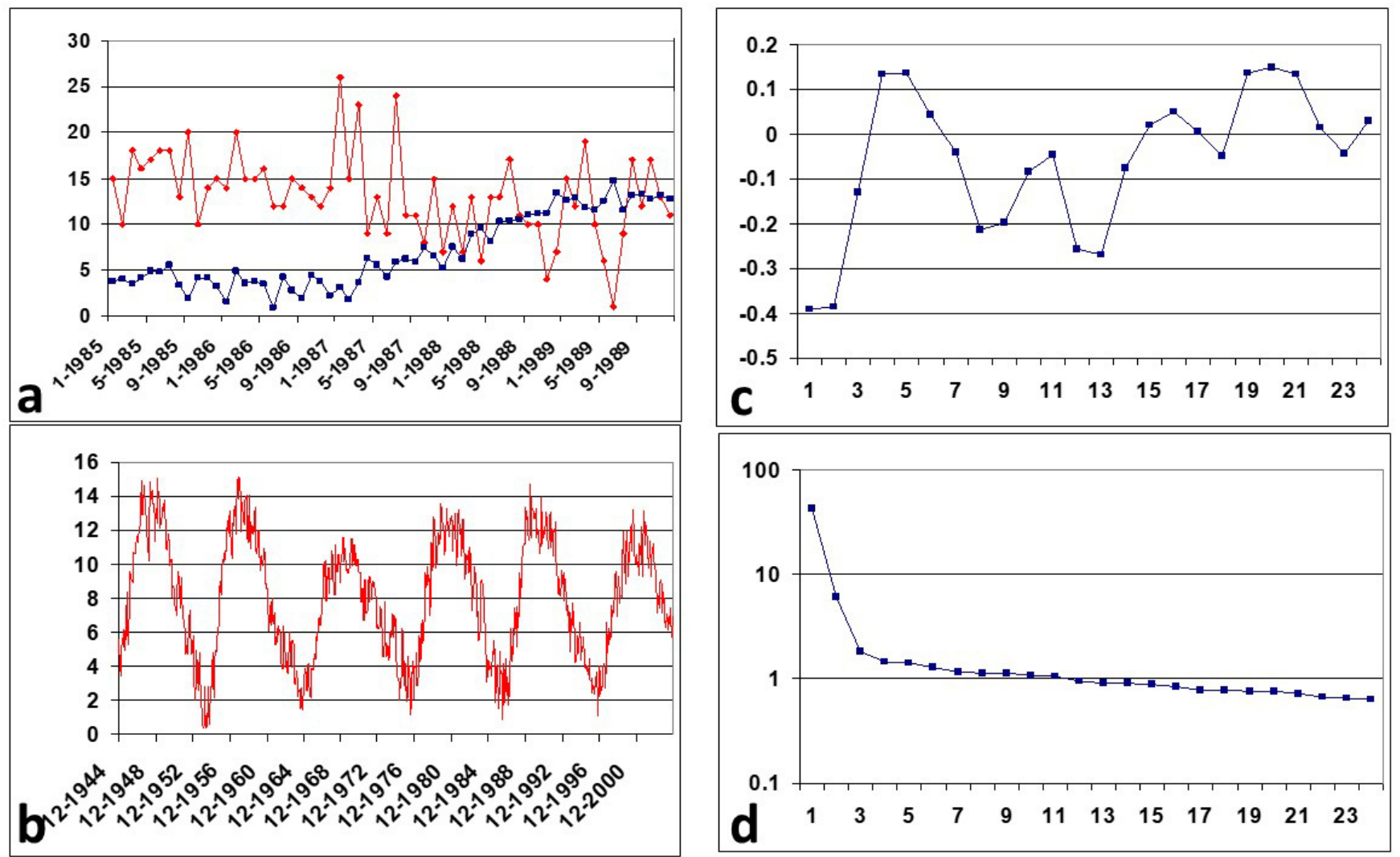

Figure 1. Singular spectrum analysis results. (a) Time series of strokes and sunspots from January 1985 to December 1989 . A square root transformation is applied to the time series of the sunspots. (b) Time series of sunspots from December 1984 to November 2004. (c) Pearson correlation coefficient between the principal components of the sunspots and strokes time series. (d) Singular values of the transformed sunspots time series. The noise plateau clearly begins at the third-ordered singular value.

pollutants. Genetic propensities also contribute to various manifestations of the chronic noncommunicable diseases. Socioeconomic and geographic disparities have been suspected, while heliomagnetic influences have been proposed as possible etiologic contributors to human pathology.

Mortality data meeting validity and credibility criteria are a sine qua non in the study of stroke incidence (Feigin \& Hoorn, 2004). Our study focused on stroke mortality in the largest Mediterranean port (Piraeus), ranked as the third most populated city in Greece. Moreover, its population is representative of the urban populations in Greece (Geronikolou, 1991). The quinquennium 1985-1989 was chosen, because, until then, Greece had a rather robust diet (low fat/sugar, proteins and vegetables/fruits daily, pure olive oil almost exclusively) and genetic homogeneity, while environmental pollution was limited. In this period, these major confounding factors were not present: major pollution, nonstandard diet, foreign gene inflow. The data used in this study were original and based on the underlined cause of death (Geronikolou, 1991). Importantly, the quinquennium selected was a period when the local population was of the same origin, while only small differences in the socioeconomically stratified levels were present. The covariates related to diet, hygiene and culture were stable in this period and, thus, they could be safely assumed.

The selected time period 1985-1989 emerged as an appropriate time to provide good reference observations, credible correlations, and future comparisons, and, hence, high inferential precision. Importantly, this period, although relatively short, included the maximum of the $22^{\text {nd }}$ cycle: July 1989 (maximum 157.6 or smoothed sunspot numbers 158.9 ), as well as the minimum of the $21^{\text {st }}$ solar cycle (minimum 13.4 or smoothed sunspot numbers 12.3). The $21^{\text {st }}$ solar cycle lasted 10.3 years, beginning in June 1976 and ending in September 1986. The 22 $2^{\text {nd }}$ solar cycle lasted 9.7 years, beginning in September 1986 and ending in May 1996. The sunspot numbers do not affect Earth directly; however, the solar wind emanating from solar activity affects stratospheric ozone layer density, whose ionization promotes health morbidity on inhabitants, including the prevalence of strokes (Feigin et al., 2014; Petropoulos \& Geronikolou, 2005).

It has been suggested that chaos and trends in local evidence are lacking (Halberg et al., 1998), and this study addresses this need. Chaotic dynamics analyses could unravel 
unknown patterns of stroke epidemiology -whose causes are not fully understood. Our work postulates that there is an inverse relation in two time series, between the timing of sunspot numbers and stroke deaths, a hypothesis posed by previous investigations (Geronikolou \& Leontisis, 2005; Geronikolou \& Petropoulos, 1996; Stoupel et al., 1999). We showed that an over $35 \%$ change in the sunspot numbers, shifted the upwards trend of stroke deaths with a delay of two months.

The interaction of living organisms and their functions with solar radiation has been previously described (Halberg et al., 1998; Reinberg et al., 2017). This consisted mainly of protein secretion studies, and, less extensively of local population dynamics. The molecular interactions network approach, where the inter-species functional interactome of nuclear steroid receptors (R1) was constructed on orthologues was employed (Geronikolou et al., 2018). R1 has interspecies dimensions and thus has evolutionary and historical value extending from insects to humans, that is, from early life eras till now. Solar activity exposure was certainly omnipresent before life appeared on the planet. Similar cycles existed, such as those detected in our study, although the rotation of our planet around its axis and around the sun were faster than they are today (Reinberg et al., 2017). R1 includes genes and their products involved in circadian rhythms, while its major hub NCOR1 in macrophages blocks the pro-atherogenic functions of peroxisome proliferatoractivated receptor gamma (PPAR $\gamma$ ) in atherosclerosis (Oppi et al., 2020), greatly implicating stroke pathophysiology. PPAR $\gamma$ has a pleiotropic role in intracerebral hemorrhage (Zhao et al., 2015) and ischemic brain injury (Culman et al., 2007). It is likely that as soon as R1 is disrupted, the atherogenic and/or other pathological processes progress dramatically, with lethal consequences. Here, both FFT and SSA revealed a novel common cycle of 6.8 days in Zurich numbers and stroke deaths. The cycle is smaller than the known anthropogenic circaseptan rhythm (Reinberg et al., 2017). The 17-ketosteroids were also found to have a $<7$ day cycle (Hamburger et al., 1985), confirming the steroid contribution to the phenomenon seen in $\mathrm{R} 1$ and herein.

It has been previously reported that the geomagnetic disturbance indices $\mathrm{K}_{\mathrm{p}}$ and aa take the value of 6.75 (Halberg et al., 1998), but without a clear association to the biota (living organisms -flora/fauna/humans). Our finding, apart from its novelty, provides a new insight in stroke epidemiology: the observed patterns suggest an endogenous natural rhythm of renewing populations.

Our work demonstrates a phase shift resulting from violent fluctuation in sunspots variation $(>35 \%)$ with a clear correlation to monthly stroke deaths. A phase delay of two months was observed between the physical triggering and the death incidence shift. This should be investigated in the future over different and/or even longer periods of time and in different and/or larger populations. Still, the violent fluctuation of $35 \%$ of sunspots appears to be a hazard for mortality and, we assume, morbidity. Thus, future medical practice should probably take account of chronopathology so as to prevent stroke mortality shifts (chronotherapy and chronoprevention plans).

\section{Conclusions}

Our work established clearly that of sunspot numbers and stroke mortality were inversely correlated, and that a violent fluctuation of sunspot numbers over $35 \%$ shifted monthly mortality to a phase delay of two months. In addition, a common, novel, non-anthropogenic chronome of 6.8 days in solar activity (sunspot numbers) and stroke mortality was revealed. Time structure patterns evaluated with non-linear methods revealed new information on the stroke epidemic, and, thus, contributed to precision inference and the need of sophisticated public health policy planning.

\section{Data availability}

Figshare: Singular Spectrum Analysis of monthly stroke deaths and mean monthly sunspot numbers. https://doi.org/10.6084/ m9.figshare.12644981.v2 (Geronikolou \& Leontitsis, 2020).

This file contains the incidence of stroke deaths by month for 1985-1989 and the incidence of sunspots by month for 1944-2004.

Data are available under the terms of the Creative Commons Attribution 4.0 International license (CC-BY 4.0).

Acknowledgements

We thank Prof Konstantinos Poulakos, Dr Mireille Harsoula and Prof Nikolaos Vogglis for their critical remarks.
Culman J, Zhao Y, Gohlke P: PPAR-gamma: therapeutic target for ischemic stroke. Trends Pharmacol Sci. 2007; 28(5): 244-249. PubMed Abstract | Publisher Full Text

Feigin V, Hoorn SV: How to study stroke incidence. Lancet. 2004; 363(9425): 1920.

PubMed Abstract | Publisher Full Text

Feigin V, Parmar PG, Barker-Collo S, et al.: Geomagnetic Storms Can Trigger StrokeEvidence From 6 Large Population-Based Studies in Europe and Australasia. Stroke. 2014; 45(6): 1639-45

PubMed Abstract | Publisher Full Text

Geronikolou S: Public and Environmental Health in Piraeus 1985-1989. Public
Hygene Bsc. University of West Attica, Athens. 1991; 300.

Reference Source

Geronikolou S, Leontisis A: Investigating internal relations of sunspot numbers and health events with SSA. 18th Summer School/Pan-Hellenic Conference: Non Linear Science and Complexity, Volos, Greece. 2005. Reference Source

Geronikolou S, Leontitsis A: Singular Spectrum Analysis of monthly stroke deaths and mean monthly sunspot numbers. figshare. Dataset. 2020. http://www.doi.org/10.6084/m9.figshare.12644981.v2

Geronikolou S, Petropoulos V: Solar activity related to geomagnetic climatic parameters and biological effects in Greece. 3rd Soltip Symposium, Beijing, 
China. 1996

Reference Source

Geronikolou S, Zikos P: Public and Environmental Health in Piraeus 1985-1989. Public Hygene. Bachelor. University of West Attica, Athens, Greece. 1991; 300. Reference Source

Geronikolou SA, Pavlopoulou A, Kanaka-Gantenbein C, et al: Inter-species functional interactome of nuclear steroid receptors (R1). Front Biosci (Elite Ed). 2018; 10: 208-228.

PubMed Abstract | Publisher Full Text

Halberg F, Siutkina EV, Cornelissen G, et al.: Chronomes render predictable the otherwise-neglected human "physiological range": position paper of the BIOCOS project. BIOsphere and the COSmos. Fiziol Cheloveka. 1998; 24(4): 14-21.

PubMed Abstract

Halgberg F, Cornelissen G, Watanabe Y, et al:: Near 10-year and longer periods modulate circadians: intersecting anti-aging and chronoastrobiological research. J Gerontol A Biol Sci Med Sci. 2001; 56(5): M304-24.

PubMed Abstract | Publisher Full Text

Hamburger C, Sothern RB, Halberg F: Circaseptans and semicircaseptan aspects of human male sexual activity. Chronobiologia. 12:250. Chronobiologia. 1985; 12: 250

Malik R, Dichgans M: Challenges and opportunities in stroke genetics.

Cardiovasc Res. 2018; 114(9): 1226-1240.

PubMed Abstract | Publisher Full Text

Mozaffarian D, Benjamin EJ, Go AS, et al. American Heart Association Statistics Committee, Stroke Statistics Subcommittee.: Heart disease and stroke statistics2015 update: a report from the American Heart Association. Circulation. 2015; 131(4): e29-322.

PubMed Abstract | Publisher Full Text

Oppi S, Nusser-Stein S, Blyszczuk P, et al.: Macrophage NCOR1 protects from atherosclerosis by repressing a pro-atherogenic PPAR $\gamma$ signature. Eur Heart $J$. 2020; 41(9): 995-1005.

PubMed Abstract | Publisher Full Text
Otsuka K, Oinuma S, Cornelissen G, et al:: Alternating light-darknessinfluenced human electrocardiographic magnetoreception in association with geomagnetic pulsations. Biomed Pharmacother. 2001; 55(Suppl 1): 63s-75s. PubMed Abstract | Publisher Full Text

Petropoulos V, Geronikolou S: Stratospheric ozon, density variation solar activity and biological phenomena. 7th HELLASET Conference Hellenic Astronomical Society, Cefalonia Greece, 2005.

Reference Source

Reinberg AE, Dejardin L, Smolensky MH, et al.: Seven-day human biological hythms: An expedition in search of their origin, synchronization, functional advantage, adaptive value and clinical relevance. Chronobiol Int. 2017; 34(2): $162-191$

PubMed Abstract | Publisher Full Text

Stoupel E, Petrauskiene J, Abramson E, et al.: Relationship between deaths from stroke and ischemic heart disease--environmental implications. J Basic Clin Physiol Pharmacol. 1999; 10(2): 135-45.

PubMed Abstract | Publisher Full Text

Stoupel E, Petrauskiene J, Kalediene R, et al.: Clinical cosmobiology: the Lithuanian study 1990-1992. Int J Biometeorol. 1995; 38(4): 204-8. PubMed Abstract | Publisher Full Text

Stoupel E, Petrauskiene J, Kalediene R, et al: Distribution of deaths from ischemic heart disease and stroke. Environmental and aging influences in men and women. J Basic Clin Physiol Pharmacol. 1996; 7(4): 303-19. PubMed Abstract | Publisher Full Text

WHO: Noncommunicable diseases. 2018

Reference Source

Yan Q: Circadian Biomarkers and Chronotherapy: Implications for Personalized and Systems Medicine. Cellular Rhythms and Networks. SpringerBriefs in Cell Biology. Springer, Cham, 2015; 71-81. Publisher Full Text

Zhao XR, Gonzales N, Aronowski J: Pleiotropic role of PPAR $\gamma$ in intracerebra hemorrhage: an intricate system involving Nrf2, RXR, and NF- $\kappa \mathrm{B}$. CNS Neurosci Ther. 2015; 21(4): 357-366.

PubMed Abstract | Publisher Full Text | Free Full Text 


\section{Open Peer Review}

\section{Current Peer Review Status:}

\section{Version 1}

Reviewer Report 13 November 2020

https://doi.org/10.5256/f1000research.27352.r70999

(C) 2020 Podolská K. This is an open access peer review report distributed under the terms of the Creative Commons Attribution License, which permits unrestricted use, distribution, and reproduction in any medium, provided the original work is properly cited.

\section{Kateřina Podolská}

Department of Ionosphere and Aeronomy, Institute of Atmospheric Physics, CAS, Prague, Czech Republic

In this manuscript, the authors propose non-anthropogenic 6.8 days cycle of stroke-related death events in Pireus port during 1985-1989 period.

Reliable data sources of solar and magnetic activity are used.

I suggest to answer the following questions and comments before the manuscript will be accepted for indexing:

Please provide codes of underlined cause of death from the of the International Classification of Diseases (ICD-9) under which was analyzed stroke-related death events registered.

Have you tried to use as another proxy of the solar activity in your analysis also Solar radio flux F10.7? The Solar radio flux reflects the nature of the process in a better way.

The discussion part should be enlarged with consideration different dynamic of geomagnetic and solar activity during the ascending and descending phase of the solar cycle. Influence of this effect in changes of mortality from cardiovascular diseases was publicized.

The effect of cosmic rays during the the solar cycle minima, to which human physiology is sensitive, should be also mentioned.

Is the work clearly and accurately presented and does it cite the current literature? Yes

Is the study design appropriate and is the work technically sound?

Yes 
Are sufficient details of methods and analysis provided to allow replication by others? Yes

If applicable, is the statistical analysis and its interpretation appropriate? Yes

Are all the source data underlying the results available to ensure full reproducibility? Yes

Are the conclusions drawn adequately supported by the results? Yes

Competing Interests: No competing interests were disclosed.

Reviewer Expertise: Stochastic Modeling, Demographic Analysis, Solar Terrestrial Interactions, Solar Flares

I confirm that I have read this submission and believe that I have an appropriate level of expertise to confirm that it is of an acceptable scientific standard.

Reviewer Report 03 November 2020

https://doi.org/10.5256/f1000research.27352.r71839

(c) 2020 Poulidakis E. This is an open access peer review report distributed under the terms of the Creative Commons Attribution License, which permits unrestricted use, distribution, and reproduction in any medium, provided the original work is properly cited.

\section{Emmanuel Poulidakis}

${ }^{1}$ Department of Cardiology, Evagelismos General Hospital, Athens, Greece

2 Hôpital Privé Saint-Martin , Caen, France

Overall it is a good work, in a rather unusual subject, but seems to well support its arguments. However, I believe that while its point is interesting, it does not sufficiently explain many of the terms used, as if addressing only those interested in the same field of research, somehow barring less expert audience. I suggest that in many cases, a few more phrases could make a difference.

For example, explaining certain terms (e.g. Sunspot numbers and time series) could make the article readable by a less specialized audience. I had to conduct research on my own to better understand these concepts. I would also suggest expanding the paragraph at the end of the introduction, detailing the aims of the study. I have attached a pdf copy with highlighted sections about possible corrections.

Another observation is the structure of the abstract, which in my opinion should be divided into sections (background/methods/results/conclusion). 
I noted a few spelling errors, highlighted in the pdf file.

Is the work clearly and accurately presented and does it cite the current literature? Yes

Is the study design appropriate and is the work technically sound?

Yes

Are sufficient details of methods and analysis provided to allow replication by others? Yes

If applicable, is the statistical analysis and its interpretation appropriate?

I cannot comment. A qualified statistician is required.

Are all the source data underlying the results available to ensure full reproducibility? Yes

Are the conclusions drawn adequately supported by the results?

Yes

Competing Interests: No competing interests were disclosed.

Reviewer Expertise: Heart failure, echocardiography, interventional cardiology.

I confirm that I have read this submission and believe that I have an appropriate level of expertise to confirm that it is of an acceptable scientific standard.

The benefits of publishing with F1000Research:

- Your article is published within days, with no editorial bias

- You can publish traditional articles, null/negative results, case reports, data notes and more

- The peer review process is transparent and collaborative

- Your article is indexed in PubMed after passing peer review

- Dedicated customer support at every stage

For pre-submission enquiries, contact research@f1000.com 